Check for updates

Cite this: RSC Adv., 2019, 9, 4336

Received 19th December 2018

Accepted 18th January 2019

DOI: $10.1039 / c 8 r a 10393 c$

rsc.li/rsc-advances

\section{Control of the dual emission from a thermally activated delayed fluorescence emitter containing phenothiazine units in organic light-emitting diodes $\dagger$}

\author{
Ikbal Marghad, (D) ab Fatima Bencheikh, ${ }^{\text {ac }}$ Chao Wang, (D) d Sophia Manolikakes, ${ }^{b}$ \\ Alice Rérat, ${ }^{\mathrm{b}}$ Corinne Gosmini, ${ }^{\mathrm{b}}$ Dae hyeon Kim, ${ }^{\mathrm{ac}}$ Jean-Charles Ribierre ${ }^{\star a c}$ \\ and Chihaya Adachi (D)*ac
}

\begin{abstract}
The development of single-component organic dual light-emitting molecules is of interest for a range of applications including white organic light-emitting diodes. Herein, a new thermally-activated delayed fluorescent molecule containing 4,6-bis-phenyl phenothiazine as donor units and 2-thiophene-1,3,5triazine as acceptor unit was synthesized using a simple cost-effective method. This compound shows two stable molecular conformations due to the presence of the phenothiazine units in its molecular structure. These conformers exhibit different photophysical properties in both solution and thin films. The electroluminescence properties of this novel emitter were then examined in organic light-emitting diodes and the results provide useful insights into the influence of the device architecture on the dual emission characteristics. The experimental results were consistent with the optical simulations and the optimized architecture led to the fabrication of electroluminescent devices with an external quantum efficiency of $11.5 \%$ and a maximum luminance value of $10370 \mathrm{~cd} \mathrm{~m}^{-2}$
\end{abstract}

\section{Introduction}

Organic light-emitting diodes (OLEDs) are now used in commercially available display applications and are extremely promising for solid-state lighting sources. Since the demonstration of fluorescent OLED in 1987 by Tang and Vanslyke, ${ }^{1}$ great enhancement of the electroluminescence (EL) quantum efficiency has been achieved using phosphorescence, ${ }^{2-4}$ triplet fusion $^{5,6}$ and thermally-activated delayed fluorescence (TADF). ${ }^{7-15}$ In particular, light-emitting molecules with TADF properties are considered now as the third generation of OLED materials and enable the harvesting of light from both singlet and triplet excitons without the use of any noble heavy metals. A large number of studies has already reported highperformance OLEDs using blue, green, red and near infrared

${ }^{a}$ Center for Organic Photonics and Electronics Research (OPERA), Kyushu University, Motooka 744, Nishi, Fukuoka 819-0395, Japan. E-mail: ribierre@opera.kyushu-u.ac. jp; adachi@opera.kyushu-u.ac.jp

${ }^{b}$ Laboratoire de chimie moléculaire LCM, Ecole Polytechnique, CNRS, 91128 Palaiseau Cedex, France

cJapan Science and Technology Agency (JST), ERATO, Adachi Molecular Exciton Engineering Project, Fukuoka 819-0395, Japan

${ }^{d}$ Department of Polymer Science and Engineering, Zhejiang University, Hangzhou 310027, China

$\dagger$ Electronic supplementary information (ESI) available: ${ }^{1} \mathrm{H}-\mathrm{NMR},{ }^{13} \mathrm{C}-\mathrm{NMR}$,

${ }^{19}$ F-NMR, quantum chemistry calculations. See DOI: $10.1039 / \mathrm{c} 8 \mathrm{ra} 10393 \mathrm{c}$
TADF emissive dopants. ${ }^{\mathbf{1 6 - 2 0}}$ In most cases, to achieve such a high-efficiency TADF emission, a small singlet-triplet energy gap $\left(\Delta E_{\mathrm{ST}}\right)$ is crucial to facilitate the upconversion from triplet to singlet excited states via thermally-activated reverse intersystem crossing (RISC) process. The most popular approach that have been used to develop TADF OLED emitters showing simultaneously small $\left(\Delta E_{\mathrm{ST}}\right)$ and increased oscillator strength is based on twisted molecular donor-acceptor architectures with large dihedral angles between the electron-donating and accepting units, resulting in a small spatial overlap between the highest occupied molecular orbital (HOMO) and the lowest unoccupied molecular orbital (LUMO) frontier orbitals. ${ }^{21-25}$ Interestingly, among the large variety of TADF OLED emitters, some of them have been found to exhibit dual emission due to the existence of stable conformers. ${ }^{26-33}$ This class of TADF emitters is evidently of strong interest for white TADF OLEDs and could be promising for other imaging or sensing applications benefiting from a broad emission. Noticeably, while the first example of single-component dual TADF emission was obtained using dyes containing phenothiazine units, ${ }^{28}$ other moieties such as 9,9-dimethyl-9,10-dihydroacridine and phenoxazine have also been recently incorporated into lightemitting dyes with dual stable conformations leading to dual TADF emission during excitations. ${ }^{26,34}$ In this context, the development of innovative TADF molecular architectures with dual conformations for highly efficient OLEDs and a deeper 
understanding of this dual emission phenomenon are still required.

In this study, we report on the synthesis of a novel TADF emitter ((T-TRZ)-PTZ) containing peripheral phenothiazine donor units and a central 2-thiophene-1,3,5-triazine acceptor unit using a cost-effective synthetic method. A schematic representation of the chemical structure of this dye is shown in Fig. 1. The 2-thiophene unit was herein chosen to shift the dual TADF emission toward longer wavelengths compared to the previously-reported phenyl analogue ${ }^{29}$ and to determine its potential influence on the electronic structure of the molecule. ${ }^{35,36}$ In this context, quantum chemistry calculations as well as the characterization of the photophysical and EL properties of this novel compound are expected to provide insights into the dual emission of TADF molecules containing phenothiazine units. In addition, optical simulations of the EL device properties are carried out to demonstrate the role played by the OLED architecture on the dual emission properties.

\section{Results and discussion}

\section{Synthesis of (T-TRZ)-PTZ}

Most of the reaction steps for the synthesis of emitters based on 1,3,5-triazine use expensive catalysts such as palladium. Recently, we reported for the first time a cost-effective and simple synthetic method for the synthesis of TADF emitters incorporating 1,3,5-triazine moieties, which is based on the cobalt catalysed cross coupling of arylzinc with aryl halide. ${ }^{29}$ The important benefit from this approach is related to its production cost which is lower than using palladium catalyst. In the present work, we used this cobalt catalysed cross coupling method for the synthesis of (T-TRZ)-PTZ 3. The synthetic route is based on three main steps as described in Scheme 1. In first place, the mono-substitution reaction of the 1,3,5-triazine with the thiophen-2-ylzinc(II) bromide catalysed by the cobalt crosscoupling was performed leading to the mono-thiophene triazine intermediate 1 containing two chloro functions that can be further substituted. This first step is then followed by another cobalt cross-coupling between the triazine intermediate 1 and the 4-fluorophenylzinc(II) bromide, previously

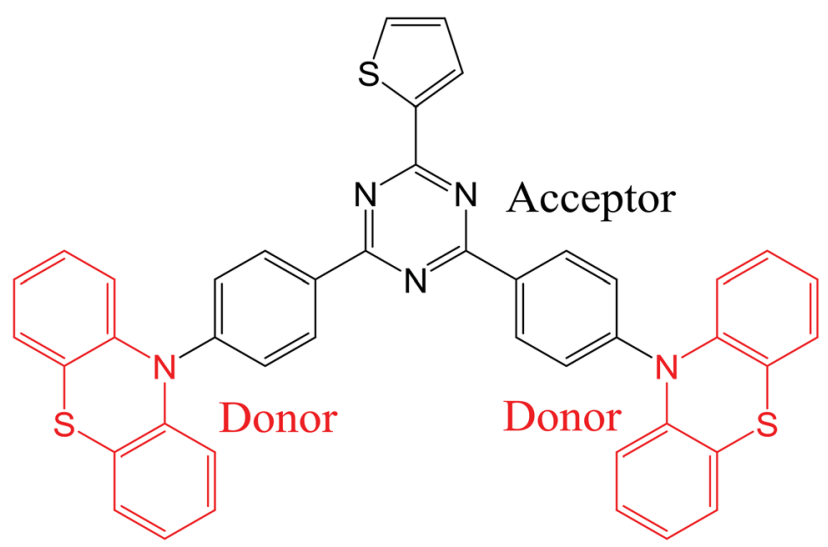

Fig. 1 Chemical structure of (T-TRZ)-PTZ. prepared from commercially available 4-bromofluorobenzene. This last coupling leads to the triazine intermediate 2 presenting two fluoro functions. The final product 3 was then obtained from 2 by a simple nucleophilic aromatic substitution of phenothiazine in presence of a strong base such as sodium hydride. Detailed chemical characterization of the intermediates and final product, including NMR and HRMS, is provided in ESI (Fig. S1-S4†).

\section{Quantum chemistry calculations}

All the quantum chemistry calculations were performed using Gaussian $09{ }^{37}$ The electronic structures of (T-TRZ)-PTZ were examined using density functional theory (DFT) and timedependent density functional theory (TDDFT) with the Tamm-Dancoff approximation (TDA) ${ }^{38}$ for avoiding triplet instabilities. ${ }^{39}$ The ground-state geometries of (T-TRZ)-PTZ were optimized at the B3LYP/6-31G* level. Because of the presence of two phenothiazine units in ((T-TRZ)-PTZ), different stable conformations can be expected for this molecule. As shown in Fig. 2 and Table S1, $\dagger$ one axial (conformer A) and one equatorial (conformer E) configurations are evidenced by the calculations and are found to show minor energy differences (Table S2 $\dagger$ ). Fig. 3 shows the distribution of the hole and electron natural transition orbitals (NTOs) of $\mathrm{S}_{1}$ and $\mathrm{T}_{1}$ calculated at the B3LYP/ 6-31G(d) level of theory based on the $S_{0}$ geometry for both conformers A and E using Multiwfn 3.4. ${ }^{40}$

For each conformer, it is interesting to note that the donor moieties dominate the hole NTOs while the acceptor moieties determine the electron NTOs. Compared to conformer A, the almost identical hole and electron NTOs for the $S_{0} \rightarrow S_{1}$ and $S_{0}$ $\rightarrow \mathrm{T}_{1}$ charge transfer (CT) transitions in conformer $\mathrm{E}$ suggest a vanishing $\Delta E_{\mathrm{ST}{ }^{41}}$ Thus a more facile exciton transformation channel via ISC and RISC may process between $S_{1}$ and $T_{1}$. As shown in Table $\mathrm{S} 3, \dagger$ the two conformers are expected to exhibit different photophysical properties. For instance, calculations indicate that the conformer A should exhibit a strong absorption peak at about $378 \mathrm{~nm}(3.28 \mathrm{eV})$ while conformer E should display a flatter and broad absorption band around $532 \mathrm{~nm}$ $(2.33 \mathrm{eV})$. The theoretical results also confirm that (T-TRZ)-PTZ should exhibit a dual emission as a direct consequence of the presence of two phenothiazine units in its molecular structure. Importantly, the theoretical results show that the calculated $\Delta E_{\mathrm{ST}}$ values of the conformers $\mathrm{A}$ and $\mathrm{E}$ in their optimized ground-state geometries are $0.46 \mathrm{eV}$ and $0.01 \mathrm{eV}$, respectively. This suggests that TADF activity should be significantly more effective in the conformer $E$. Interestingly, these $\Delta E_{\mathrm{ST}}$ values are slightly different from those calculated in the previouslyreported phenyl analogue ${ }^{29}(0.0079$ and $0.71 \mathrm{eV}$ for the $\mathrm{E}$ and A conformers, respectively).

\section{Photophysical properties of (T-TRZ)-PTZ}

The absorption and steady-state photoluminescence (PL) spectra of (T-TRZ)-PTZ measured at room temperature in degassed toluene solution are displayed in Fig. 4a. Concentration of the solution was $1 \times 10^{-5} \mathrm{M}$. The dye (T-TRZ)-PTZ exhibits two main intense absorption bands at 330 and 

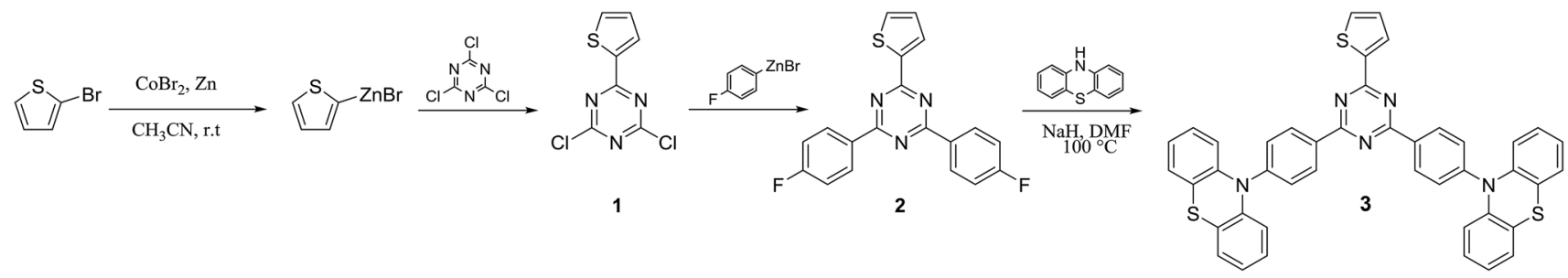

Scheme 1 Synthesis of (T-TRZ)-PTZ.

$364 \mathrm{~nm}$, which are assigned to the ${ }_{1}\left(\pi, \pi^{*}\right) \leftarrow \mathrm{S}_{0}$ transition of the $1,3,5$ triazine. In addition, the weak absorption tail at wavelengths longer than $400 \mathrm{~nm}$ is related to the intramolecular CT character of the quasi-equatorial conformer of the molecule. The steady-state PL spectrum of (T-TRZ)-PTZ in toluene shows a dual emission with two peaks at around 430 and $593 \mathrm{~nm}$, respectively. The peak at $593 \mathrm{~nm}$ shows the highest intensity, a large Stokes shift and a full-width-at-half-maximum of $96 \mathrm{~nm}$, which can be assigned to the emission from the singlet excited state of the quasi-equatorial conformation. The peak at $430 \mathrm{~nm}$ presents a significantly lower PL intensity and is attributed to the emission from the singlet excited state of the quasi-axial conformer. Noticeably, the PL emission of (T-TRZ)-PTZ in toluene is about $18 \mathrm{~nm}$ red-shifted compared to that of the previously-reported phenyl analogue. ${ }^{29}$ The results indicate that the incorporation of the thiophene unit in (T-TRZ)-PTZ affects the singlet-triplet gap as evidenced by the quantum chemistry calculations and the steady-state PL emission of the molecule.

Fig. 4b shows the absorption and steady-state PL spectra of (T-TRZ)-PTZ doping at $6 \mathrm{wt} \%$ a $3,3^{\prime}$-bis( $N$-carbazolyl)-1,1'biphenyl (mCBP) host. The film was thermally evaporated on top of a fused silica substrate. The mCBP host was selected due to its high triplet energy $\mathrm{T}_{1}$, which prevents back energy transfer from the guest to the host molecules and confines the triplets within the guest emitters. Because of the small doping concentration of the guest molecules, the absorption spectrum of the mCBP blend is strongly dominated by the absorption of the host molecules. In particular, the absorption bands observed at 330 and $344 \mathrm{~nm}$ are due to the presence of carbazole units in the mCBP molecule. ${ }^{42}$ Noticeably, considering the excitation wavelength of $340 \mathrm{~nm}$, the steady-state PL spectrum of the mCBP blend does not show any emission from the host.
This implies that an efficient energy transfer takes place from the mCBP host to the (T-TRZ)-PTZ doping molecules. ${ }^{43-45}$ It can also be seen that the steady-state PL spectrum of the blend film exhibits a dual emission with two peaks located around 423 and $523 \mathrm{~nm}$, respectively. Similarly to the solution case, the low and high energy peaks are attributed to the quasi-equatorial and quasi-axial conformers, respectively. It is worth noting that the emission peak of the quasi-equatorial conformer is significantly blue-shifted in the blend, as compared to its PL spectrum in toluene. This is presumably due to the ICT character of (T-TRZ)PTZ and the different polarity of the environment. ${ }^{11,20,46,47}$

To gain further insights into the photophysics of (T-TRZ)PTZ, the transient PL decay of the equatorial conformer was measured in degased and non-degased toluene solution at a low concentration around $10^{-6} \mathrm{M}$. Excitation wavelength was $337 \mathrm{~nm}$ and the emission wavelength was $593 \mathrm{~nm}$. The kinetics shown in Fig. 5 can be described by the sum of two exponential decay functions. We define the slow and fast time constants characteristics of these exponential decay functions to the delayed and prompt fluorescence lifetimes, respectively. While the prompt fluorescence lifetime does not change with the presence or not of oxygen and is in the order of $10 \mathrm{~ns}$, the delayed fluorescence lifetime is found to increase from $50 \mathrm{~ns}$ to $570 \mathrm{~ns}$ after degasing the toluene solution. These data indicate that the equatorial conformer of (T-TRZ)-PTZ shows some TADF properties, in accordance with the theoretical results obtained by quantum chemistry calculations. The observed behavior in presence of oxygen shows indeed that the triplet states involved in the TADF process are readily quenched by the oxygen triplet ground state and this fully supports the assignment of TADF. An important parameter which needs to be measured in order to evaluate the potential of (T-TRZ)-PTZ for OLEDs is its
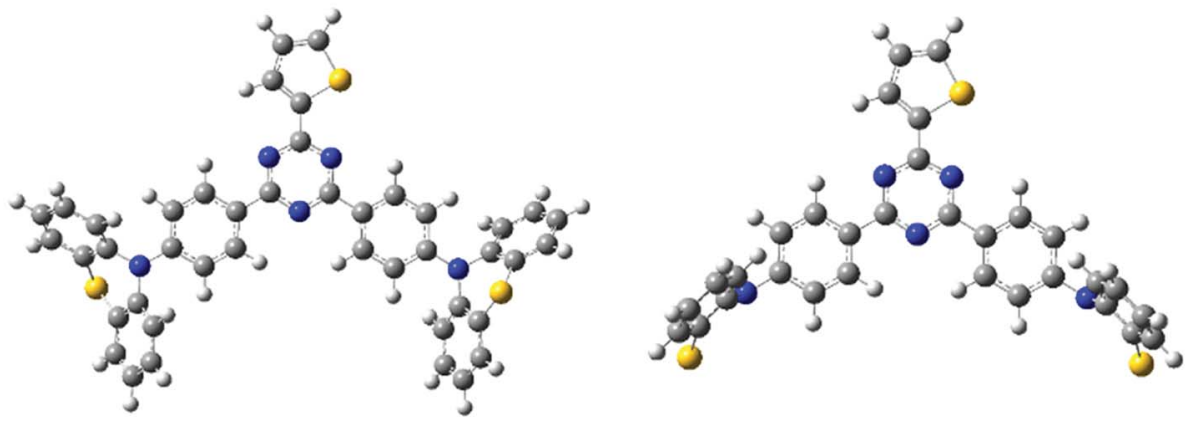

Fig. 2 Molecular geometries of the conformers A (left) and E (right) in their ground-state optimized at the B3LYP/6-31G(d) level of theory. Note that only half of the phenothiazine units can be seen in this representation of the conformer $E$. 

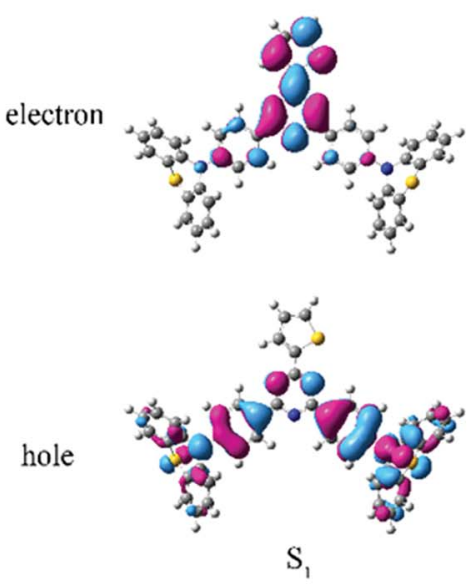

Isomer A
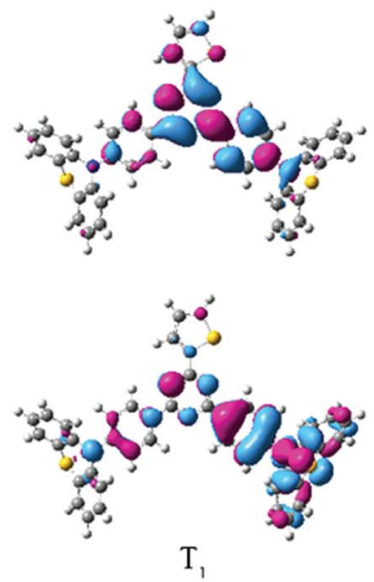
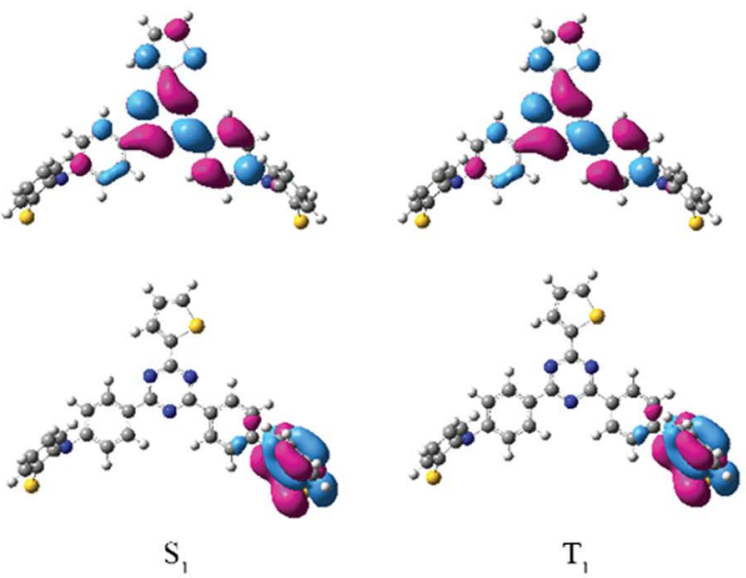

Isomer $\mathrm{E}$

Fig. 3 Distribution of the hole and electron NTOs of $S_{1}$ and $T_{1}$ calculated at the B3LYP/6-31G(d) level of theory based on the $S_{0}$ geometry for the conformers $A$ and $E$. Note that only half of the phenothiazine units can be seen in the representation of the conformers $E$.
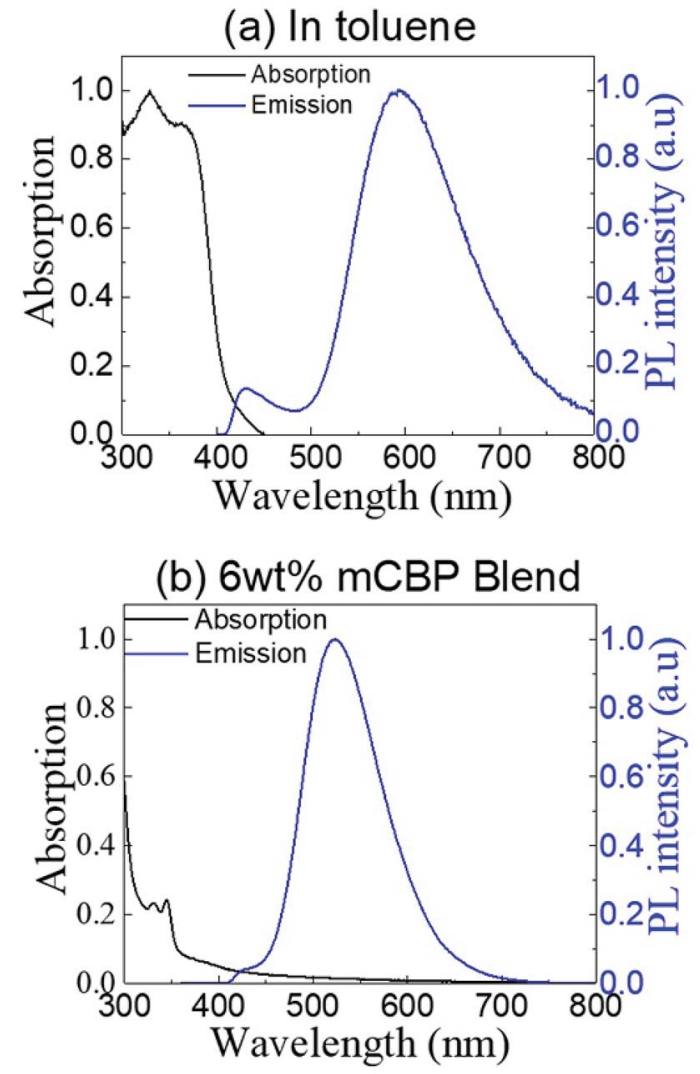

Fig. 4 Normalized absorption (black line) and steady-state photoluminescence (blue line) spectra of (T-TRZ)-PTZ (a) in degassed toluene solution with a concentration of $1 \times 10^{-5} \mathrm{M}$ and (b) in $6 \mathrm{wt} \%$ $\mathrm{mCBP}$ blend. The emission spectra were obtained using an excitation wavelength of $340 \mathrm{~nm}$.

photoluminescence quantum yield (PLQY) in thin film. We found that the PLQY of the $6 \mathrm{wt} \% \mathrm{mCBP}$ blend in nitrogen atmosphere yields a value of $43 \%$.

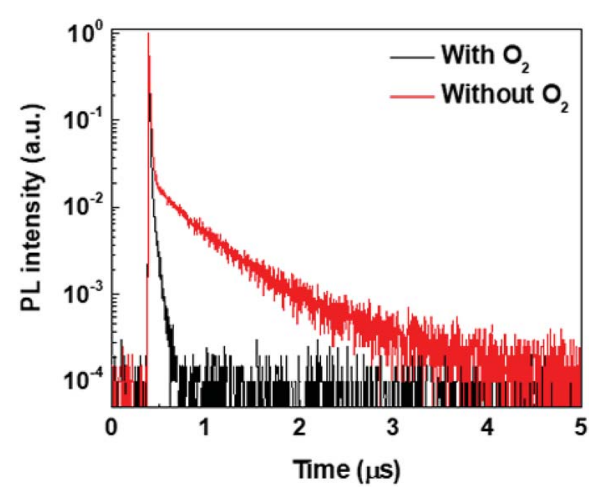

Fig. 5 Transient fluorescence decays of (T-TRZ)-PTZ in degased and non-degased toluene solutions. Excitation wavelength was $337 \mathrm{~nm}$ and emission wavelength was $593 \mathrm{~nm}$.

\section{Characterization of the OLEDs}

To examine the EL properties of (T-TRZ)-PTZ, the first batch of OLEDs had the following architecture: indium tin oxide (ITO) (100 nm)/ $N, N^{\prime}$-diphenyl- $N, N^{\prime}$-bis(1-naphthyl)-1,10-biphenyl-4, $4^{\prime}$ diamine $(\alpha$-NPD) (20 $\mathrm{nm}) /$ tris(4-carbazoyl-9-ylphenyl)amine (TCTA) (10 nm)/1,3-bis( $N$-carbazoyl)benzene (mCP) $(10 \mathrm{~nm}) /$ $6 \mathrm{wt} \%$ (T-TRZ)-PTZ: mCBP (15 nm)/bis[2-(diphenylphosphino) phenyl]ether oxide (DPEPO) (10 nm)/1,3,5-tris(2- $N$-phenylbenzimidazolyl)benzene (TPBi) $(55 \mathrm{~nm}) /$ lithium fluoride (LiF) $(0.8 \mathrm{~nm})$ /aluminium (Al) $(900 \mathrm{~nm})$. A schematic representation and energy diagram of the device structure are provided in Fig. 6a. $\alpha$-NPD and MCP were chosen for the hole transport layers while DPEPO and TPBi were selected for the hole blocking and electron transport layers, respectively. Fig. 6b-d show the EL characteristics of this OLED. The device exhibits greenyellowish emission with a turn-on voltage of $4.6 \mathrm{~V}$, a maximum current efficiency of $35.47 \mathrm{~cd} \mathrm{~A}^{-1}$, a power efficiency of $11.1 \mathrm{~lm} \mathrm{~W}^{-1}$, a maximum luminance up to $10370 \mathrm{~cd} \mathrm{~m}^{-2}$ and a maximum EQE of $9.4 \%$ without any light outcoupling 
a)

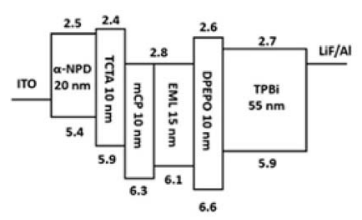

c)

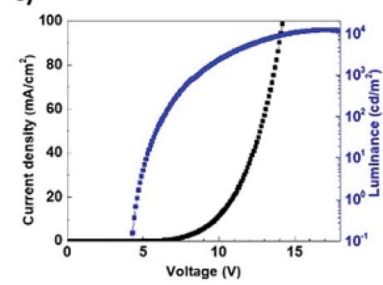

b)

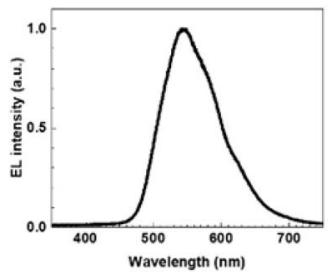

d)

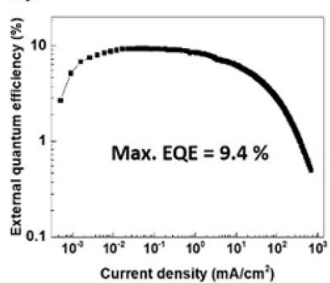

Fig. 6 (a) Schematic representation and energy diagram of the OLED structure. (b) Electroluminescence spectra at different current densities. (c) Current density-voltage-luminance characteristic and (d) external quantum efficiency as a function of the current density.

enhancement. These data could be reproduced in several devices. The obtained EQE value is significantly higher than the upper theoretical limit stated for conventional fluorescent OLEDs. We attribute this result to an efficient TADF process enabling the effective upconversion of the triplets to the singlets via RISC, $^{7}$ which is in agreement with the quantum chemistry calculations and previous reports devoted to similar dyes.

Looking at the EL spectra in Fig. 6b, it can be seen that only the emission from the quasi-equatorial conformer is observed. Previous reports have provided evidence of the strong influence of the OLED architecture on the EL spectra due to optical cavity effects. ${ }^{4-53}$ In order to examine the potential impact of these optical cavity effects on the dual emission spectrum of (T-TRZ)PTZ, we carried out optical simulations to see if these optical cavity effects could hide the emission band at $427 \mathrm{~nm}$. These optical simulations were performed using SETFOS 4.2 software in the wavelength range between 380 and $750 \mathrm{~nm}$. The lightemitting process taking place in the emissive layer of the OLED is simulated based on the model of emissive dipoles embedded within a multilayer stack. ${ }^{54}$ Since the thicknesses of these layers are comparable to the wavelength of the emitted light, internal reflections at the different interfaces occur, leading to optical interference effects. To model these interference effects in the OLED, a transfer matrix method is used, ${ }^{55}$ taking into account the optical indices of the emissive layer determined by variable angle spectroscopic ellipsometry. ${ }^{56}$ It should be noted that the calculation was carried out by assuming randomly distributed transition dipole moment approximated by a Dirac distribution placed at the interface between the emissive layer and the mCBP one.

It is well established that the distance between the emitting zone and the metal cathode predominantly affects the interference conditions at the position of the emissive zone. In this context, we decided to vary the thickness of the TPBi layer in the (T-TRZ)-PTZ OLED and see how this affects the intensity of the band at $427 \mathrm{~nm}$ in the EL spectrum. Fig. 7 shows the calculated

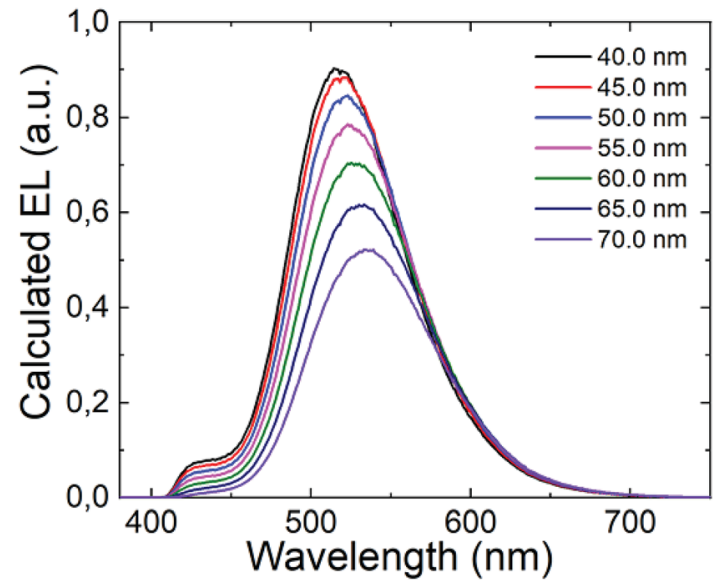

Fig. 7 Calculated EL spectra of the (T-TRZ)-PTZ OLED with different thicknesses of TPBi.

EL spectrum of the OLED for different thicknesses of the TPBi film (varying from 40 to $70 \mathrm{~nm}$ ). The results demonstrate that the EL spectrum is red-shifted and the intensity of the band at $427 \mathrm{~nm}$ is decreased when the TPBi thickness increases. In other words, the fact that the emission peak at $427 \mathrm{~nm}$ was not

a)
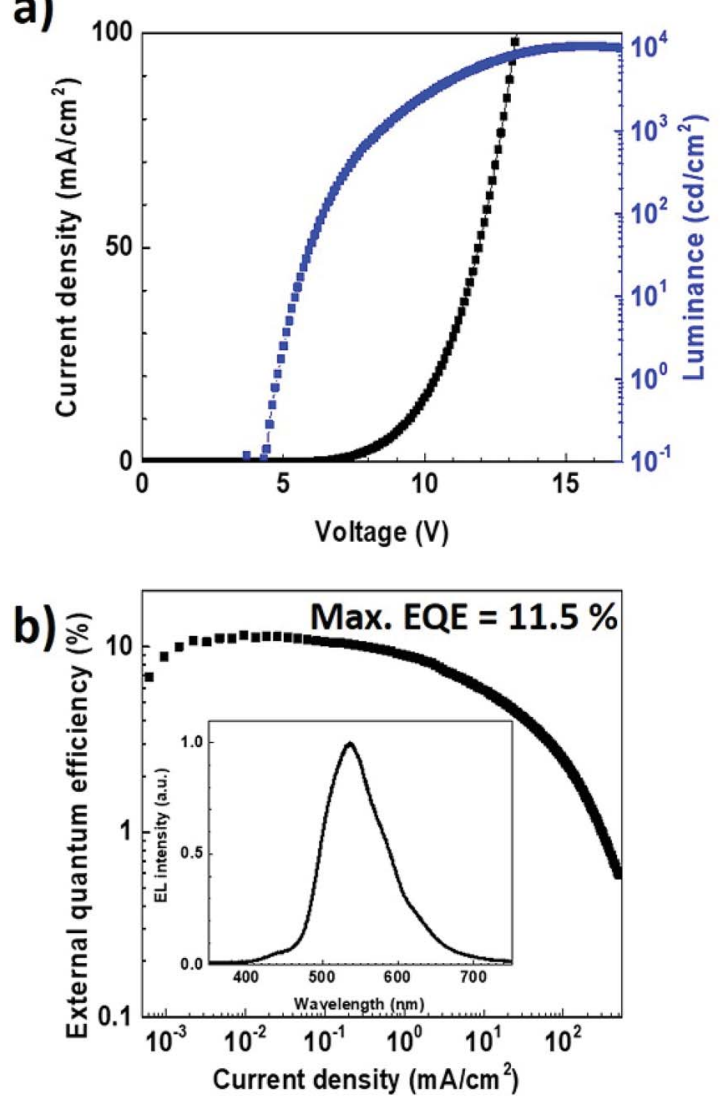

Fig. 8 (a) Current density-voltage-luminance characteristic and (b) external quantum efficiency as a function of the current density for the OLED with a TPBi film thickness of $35 \mathrm{~nm}$. The electroluminescence spectrum obtained for a current density of $100 \mathrm{~mA} \mathrm{~cm}^{-2}$ in shown in the inset. 
observed in the EL spectrum can be explained by these interference effects. This clearly demonstrates that the architecture of the OLED should be carefully designed to optimize the dual emission in the EL spectrum.

To verify experimentally these modelling results, we fabricated an OLED using the same multilayer structure but with a thickness of $35 \mathrm{~nm}$ for the TPBi layer. The electroluminescence data obtained with such a device architecture are reported in Fig. 8. Noticeably, in contrast to what was achieved with a TPBi film thickness of $55 \mathrm{~nm}$, the emission peak at $427 \mathrm{~nm}$ can be observed in the EL spectrum. This fully supports the results obtained from the optical simulations and demonstrates the importance of the optical cavity effects on the dual emission from TADF OLEDs. The $J-V$ curve and the EQE of the TADF OLED were also measured as a function of the current density. The maximum EQE value and luminance measured for this OLED were found to be as high as $11.5 \%$ and $10370 \mathrm{~cd} \mathrm{~cm}^{-2}$, respectively, providing evidence that not only the dual emission but also the overall performances of the EL device was improved after the optimization of the TPBi film thickness.

\section{Conclusions}

In summary, we designed and synthesized the novel TADF molecule (T-TRZ)-PTZ, which contains 4,6-bis-phenyl phenothiazine as donor units and 2-thiophene-1,3,5-triazine as acceptor unit. A simple and low-cost synthetic method based on cobalt catalysed cross-coupling was used to produce this lightemitting compound. This method fits with the asset of reducing OLED cost thanks to the used low-cost reagents. The photoluminescence spectrum of (T-TRZ)-PTZ in solution and film exhibits a dual emission which is due to the incorporation of phenothiazine unit in the molecular structure, resulting in the existence of two stable molecular conformations for ( $\mathrm{T}$ TRZ)-PTZ. The electroluminescence properties of (T-TRZ)-PTZ are then characterized in OLEDs and the best device exhibited maximum external quantum efficiency and luminance values of $11.5 \%$ and $10370 \mathrm{~cd} \mathrm{~m}^{-2}$, respectively. Based on experimental and optical simulations results, this study also provides new useful evidences that the OLED architecture must be carefully designed to optimize the dual emission in the electroluminescence spectrum. This latter result should be extremely relevant to further studies devoted to the development of high-efficiency white OLEDs based on single-component organic dual lightemitting molecules.

\section{Experimental section}

\section{General information}

All chemicals are reagent/analytical grade and used without further purification. Chromatographic purification of the products was accomplished using forced-flow chromatography on Geduran Silica Gel 60 40-63 mesh according to the method of Still. Thin-layer chromatography (TLC) was performed on Merck $0.20 \mathrm{~mm}$ silica gel 60-F254 plates. Visualisation of the developed chromatogram was performed by using UV light $(254 \mathrm{~nm}) .{ }^{1} \mathrm{H},{ }^{13} \mathrm{C},{ }^{19} \mathrm{~F}$ NMR spectra were recorded on Bruker AC-300 (300 MHz, $75 \mathrm{MHz}$ and $282 \mathrm{MHz}$ respectively) as noted, and are internally referenced to residual solvent signals $\left(\mathrm{CDCl}_{3}\right)$. Data for ${ }^{1} \mathrm{H},{ }^{13} \mathrm{C}$ and ${ }^{19} \mathrm{~F}$ NMR are reported as follows: chemical shift $(\delta \mathrm{ppm})$, multiplicity ( $\mathrm{s}=$ singlet, $\mathrm{d}=$ doublet, $\mathrm{t}=$ triplet, $\mathrm{q}=$ quadruplet, $\mathrm{m}=$ multiplet), coupling constant and integration. High resolution mass spectra were obtained from the Ecole Polytechnique; Mass Spectral facility. Melting points were determined with a Stuart Automatic Melting Point SMP 40 and are uncorrected. All of the starting materials were purchased from the usual suppliers (Sigma-Aldrich, Alfa Aesar, and Acros Organics).

\section{Synthesis}

2,4-Dichloro-6-(thiophen-2-yl)-1,3,5-triazine. $\mathrm{CoBr}_{2}$ (437 mg, $2 \mathrm{mmol}, 0.1$ equiv.) and $\mathrm{Zn}$ dust ( $3.9 \mathrm{~g}, 60 \mathrm{mmol}, 3$ equiv.) were added in MeCN (16 mL). The mixture was stirred at room temperature for 1 hour and 2-bromothiophene $(1.92 \mathrm{~mL}$, $20 \mathrm{mmol}, 1$ equiv.) was added and the reaction was stirred at room temperature for 6 hours. The reaction was stirred to give the thiophen-2-ylzinc(II) bromide reagent (100\% in GC). 2,4,6Trichloro-1,3,5-triazine ( $4.43 \mathrm{~g}, 24 \mathrm{mmol}, 1.2$ equiv.) was added. The mixture was stirred for $18 \mathrm{~h}$ at room temperature. The reaction was quenched by addition of saturated aqueous $\mathrm{NH}_{4} \mathrm{Cl}$ solution $(80 \mathrm{~mL})$ followed by extraction with dichloromethane $(4 \times 100 \mathrm{~mL})$. The combined organic layers were dried over $\mathrm{MgSO}_{4}$ and after filtration the solvents were evaporated in vacuum. Recrystallization from methanol and ethyl acetate afforded the product in $54 \%(2.4 \mathrm{~g})$ yield as a yellow solid.

${ }^{1} \mathrm{H}-\mathrm{NMR}\left(300 \mathrm{MHz}, \mathrm{CDCl}_{3}\right): \delta / \mathrm{ppm}=8.27(\mathrm{dd}, J=3.9,1.2 \mathrm{~Hz}$, $1 \mathrm{H}), 7.77$ (dd, $J=5.0,1.2 \mathrm{~Hz}, 1 \mathrm{H}), 7.25-7.21(\mathrm{~m}, 1 \mathrm{H}) .{ }^{13} \mathrm{C}-\mathrm{NMR}$ $\left(75 \mathrm{MHz}, \mathrm{CDCl}_{3}\right): \delta / \mathrm{ppm}=171.8,170.3,138.1,136.3,135.2$, 129.4. HRMS (EI+) $\left(\mathrm{C}_{7} \mathrm{H}_{3} \mathrm{Cl}_{2} \mathrm{~N}_{3} \mathrm{~S}\right)$ : calculated $\mathrm{m} / \mathrm{z}: 230.9425$, found: 230.5421 .

2,4-Bis(4-fluorophenyl)-6-(thiophen-2-yl)-1,3,5-triazine. $\mathrm{CoBr}_{2}$ (109 mg, $0.5 \mathrm{mmol}, 0.1$ equiv.) and zinc dust (1.08 g, $16.5 \mathrm{mmol}$, 3.3 equiv.) were added in acetonitrile $(4 \mathrm{~mL})$. The mixture was stirred at room temperature for $1 \mathrm{~h}$ and 4-bromofluorobenzene ( $0.55 \mathrm{~mL}, 5 \mathrm{mmol}, 1$ equiv.) was added and the reaction was stirred at room temperature for 1 hour to give the (4-fluorophenyl)zinc bromide reagent (80\% in GC). 2,4-Dichloro-6(thiophen-2-yl)-1,3,5-triazine was added (274 mg, $1.19 \mathrm{mmol}$, 0.35 equiv.). The mixture was stirred for $18 \mathrm{~h}$ at room temperature. The reaction was quenched by addition of saturated aqueous $\mathrm{NH}_{4} \mathrm{Cl}$ solution $(40 \mathrm{~mL})$ followed by extraction with dichloromethane $(4 \times 40 \mathrm{~mL})$. The combined organic layers were dried over $\mathrm{MgSO}_{4}$ and after filtration the solvents were evaporated in vacuum. Recrystallization from methanol and ethyl acetate afforded the product in $60 \%(210 \mathrm{mg})$ yield as a white solid.

${ }^{1} \mathrm{H}-\mathrm{NMR}\left(300 \mathrm{MHz}, \mathrm{CDCl}_{3}\right): \delta / \mathrm{ppm}=8.73(\mathrm{dd}, J=9.0,5.6 \mathrm{~Hz}$, $4 \mathrm{H}), 8.35(\mathrm{dd}, J=3.8,1.2 \mathrm{~Hz}, 1 \mathrm{H}), 7.66(\mathrm{dd}, J=5.0,1.2 \mathrm{~Hz}, 1 \mathrm{H})$, 7.27-7.20 (m, 5H). ${ }^{13} \mathrm{C}-\mathrm{NMR}\left(75 \mathrm{MHz}, \mathrm{CDCl}_{3}\right): \delta / \mathrm{ppm}=170.7$, $168.2,166.0(\mathrm{~d}, J=253.2 \mathrm{~Hz}), 142.0,132.5,132.1(\mathrm{~d}, J=2.9 \mathrm{~Hz})$, 131.8, 131.4 (d, $J=9.1 \mathrm{~Hz}), 128.7,115.9$ (d, $J=21.8 \mathrm{~Hz}) .{ }^{19} \mathrm{~F}-$ NMR $\left(282 \mathrm{MHz}, \mathrm{CDCl}_{3}\right): \delta / \mathrm{ppm}=-106.81$. HRMS (EI+) $\left(\mathrm{C}_{19} \mathrm{H}_{11} \mathrm{~F}_{2} \mathrm{~N}_{3} \mathrm{~S}\right)$ : calculated $\mathrm{m} / z$ : 351.0642 , found: 351.0641 . 
10,10'-((6-(Thiophen-2-yl)-1,3,5-triazine-2,4-diyl)bis $(4,1-$ phenylene) $)$ bis( $10 \mathrm{H}$-phenothiazine). To a solution of phenothiazine (52 mg, $0.26 \mathrm{mmol}, 2.2$ equiv.) in dry DMF under nitrogen was added sodium hydride $(20 \mathrm{mg}, 60 \%$ dispersion in mineral oil, $0.53 \mathrm{mmol}, 4.4$ equiv.). The reaction mixture is stirred at room temperature for 2 hours, a solution of 2,4-bis(4fluorophenyl)-6-(thiophen-2-yl)-1,3,5-triazine $(41 \quad \mathrm{mg}$, $0.12 \mathrm{mmol}, 1$ equiv.) in $1 \mathrm{~mL}$ of DMF was added and the mixture is stirred at $100{ }^{\circ} \mathrm{C}$ for 18 hours. The reaction was quenched by addition of water $(10 \mathrm{~mL})$ followed by extraction with ethyl acetate $(3 \times 10 \mathrm{~mL})$. The combined organic layers were washed with a $1 \mathrm{M}$ aqueous lithium chloride solution (3 $\times 20 \mathrm{~mL})$, then with a $2 \mathrm{M}$ aqueous $\mathrm{NaOH}$ solution $(3 \times 20$ $\mathrm{mL}$ ), dried over $\mathrm{MgSO}_{4}$ and after filtration the solvents were evaporated in vacuum. Recrystallization from ethyl acetate and petroleum ether afforded the product in 54\% (46 mg) yield as a yellow solid.

${ }^{1} \mathrm{H}-\mathrm{NMR}\left(300 \mathrm{MHz}, \mathrm{CDCl}_{3}\right): \delta / \mathrm{ppm}=8.79(\mathrm{~d}, J=8.6 \mathrm{~Hz}, 4 \mathrm{H})$, $8.36(\mathrm{~d}, J=3.7 \mathrm{~Hz}, 1 \mathrm{H}), 7.65(\mathrm{dd}, J=5.0,1.2 \mathrm{~Hz}, 1 \mathrm{H}), 7.44(\mathrm{~d}, J=$ $8.6 \mathrm{~Hz}, 4 \mathrm{H}), 7.26-7.24(\mathrm{~m}, 1 \mathrm{H}), 7.21(\mathrm{dd}, J=7.5,1.6 \mathrm{~Hz}, 4 \mathrm{H}), 7.06$ $(\mathrm{td}, J=7.5,1.6 \mathrm{~Hz}, 4 \mathrm{H}), 6.98(\mathrm{td}, J=7.5,1.2 \mathrm{~Hz}, 4 \mathrm{H}), 6.74(\mathrm{dd}, J$ $=7.5,10.2 \mathrm{~Hz}, 4 \mathrm{H}) .{ }^{13} \mathrm{C}-\mathrm{NMR}\left(75 \mathrm{MHz}, \mathrm{CDCl}_{3}\right): \delta / \mathrm{ppm}=170.9$, 168.1, 146.8, 143.1, 142.2, 133.1, 132.4, 131.6, 131.2, 128.7, $127.7,127.2,125.7,125.2,124.1,120.3$. HRMS (EI+) $\left(\mathrm{C}_{43} \mathrm{H}_{27} \mathrm{~N}_{5} \mathrm{~S}_{3}\right)$ : calculated $\mathrm{m} / z$ : 709.1429, found: 709.1416.

\section{Photophysical measurements}

For the photophysical measurements, the degassed toluene solution of (T-TRZ)-PTZ was placed in a quartz cuvette. In parallel, the organic thin film was deposited by thermal evaporation onto a precleaned fused silica substrate. UVvisible absorption and steady-state PL spectra were measured using a UV-2550 spectrometer (Shimadzu) and a Fluoromax-4 spectrophotometer (Horiba Scientific), respectively. The transient PL decay was measured by a streak camera (Hamamatsu Photonics C4334). The samples were placed under vacuum $(<4$ $\times 10^{-1} \mathrm{~Pa}$ ) and photoexcited by a nitrogen gas laser (Ken-X, Usho Optical Systems) delivering 500 ps pulses at a repetition rate of $20 \mathrm{~Hz}$ and emitting at $337 \mathrm{~nm}$.

\section{OLED fabrication and measurements}

The devices were prepared using precleaned indium tin oxide (ITO) substrates. The different organic layers were sequentially thermally evaporated under vacuum lower than $3 \times 10^{-4} \mathrm{~Pa}$. Finally, the LiF/Al electrodes were deposited through a shadow mask on top of the multilayer structure. The device active area was $4 \mathrm{~mm}^{2}$. To avoid any degradation and emission quenching due to oxygen and moisture, the OLEDs were encapsulated in a glovebox filled with nitrogen. The $J-V-L$ characteristics were collected using a source meter (Keithley 2400, Keithley Instruments Inc.) and an absolute external quantum efficiency measurement system (C9920-12, Hamamatsu Photonics). An optical fiber connected to a spectrometer (PMA-12, Hamamatsu Photonics) was used to record the electroluminescence spectra.

\section{Conflicts of interest}

There are no conflicts to declare.

\section{Acknowledgements}

This work was supported by JST ERATO Grant Number JPMJER1305, Japan.

\section{References}

1 C. W. Tang and S. A. VanSlyke, Appl. Phys. Lett., 1987, 51, 913-915.

2 S. R. Forrest, M. A. Baldo, D. F. O'Brien, Y. You, A. Shoustikov, S. Sibley and M. E. Thompson, Nature, 1998, 395, 151-154.

3 K. H. Kim, S. Lee, C. K. Moon, S. Y. Kim, Y. S. Park, J. H. Lee, J. Woo Lee, J. Huh, Y. You and J. J. Kim, Nat. Commun., 2014, $5,4769$.

4 J. C. Ribierre, S. G. Stevenson, I. D. W. Samuel, S. V. Staton and P. L. Burn, J. Disp. Technol., 2007, 3, 233-237.

5 C. J. Chiang, A. Kimyonok, M. K. Etherington, G. C. Griffiths, V. Jankus, F. Turksoy and A. P. Monkman, Adv. Funct. Mater., 2013, 23, 739-746.

6 J. Xue, C. Li, L. Xin, L. Duan and J. Qiao, Chem. Sci., 2016, 7, 2888-2895.

7 H. Uoyama, K. Goushi, K. Shizu, H. Nomura and C. Adachi, Nature, 2012, 492, 234-238.

8 A. Endo, M. Ogasawara, D. Takahashi, D. Yokoyama, Y. Kato and C. Adachi, Adv. Mater., 2009, 21, 4802-4805.

9 D. H. Kim, K. Inada, L. Zhao, T. Komino, N. Matsumoto, J. C. Ribierre and C. Adachi, J. Mater. Chem. C, 2017, 5, 1216.

10 M. Y. Wong and E. Zysman-Colman, Adv. Mater., 2017, 29, 1605444.

11 Y. Hao, D. H. Kim, X. K. Chen, A. S. D. Sandanayaka, J. U. Kim, E. Zaborova, G. Canard, Y. Tsuchiya, E. Y. Choi, J. W. Wu, F. Fages, J. L. Bredas, A. D'Aleo, J. C. Ribierre and C. Adachi, Chem. Mater., 2018, 30, 6702.

12 D. Zhang, M. Cai, Y. Zhang, D. Zhang and L. Duan, Mater. Horiz., 2016, 3, 145-151.

13 H. Wang, L. Xie, Q. Peng, L. Meng, Y. Wang, Y. Yi and P. Wang, Adv. Mater., 2014, 26, 5198-5204.

14 F. B. Dias, K. N. Bourdakos, V. Jankus, K. C. Moss, K. T. Kamtekar, V. Bhalla, J. Santos, M. R. Bryce and A. P. Monkman, Adv. Mater., 2013, 25, 3707-3714.

15 T. A. Lin, T. Chatterjee, W. L. Tsai, W. K. Lee, M. J. Wu, M. Jiao, K. C. Pan, C. L. Yi, C. L. Chung and K. T. Wong, Adv. Mater., 2016, 28, 6976-6983.

16 Q. Zhang, B. Li, S. Huang, H. Nomura, H. Tanaka and C. Adachi, Nat. Photonics, 2014, 8, 326-332.

17 H. Kaji, H. Suzuki, T. Fukushima, K. Shizu, K. Suzuki, S. Kubo, T. Komino, H. Oiwa, F. Suzuki, A. Wakamiya, Y. Murata and C. Adachi, Nat. Commun., 2015, 6, 8476.

18 S. Hirata, Y. Sakai, K. Masui, H. Tanaka, S. Y. Lee, H. Nomura, N. Nakamura, M. Yasumatsu, H. Nakanotani, Q. Zhang, K. Shizu, H. Miyazaki and C. Adachi, Nat. Mater., 2015, 14, 330-336. 
19 Q. Zhang, H. kuwabara, W. J. Postcavage, S. Huang, Y. Hatae, T. Shibata and C. Adachi, J. Am. Chem. Soc., 2014, 136, 18070-18081.

20 D. H. Kim, A. D'Aleo, X. K. Chen, A. D. S. Sandanayaka, D. D. Yao, L. Zhao, T. Komino, E. Zaborova, G. Canard, Y. Tsuchiya, E. Y. Choi, J. W. Wu, F. Fages, J. L. Bredas, J. C. Ribierre and C. Adachi, Nat. Photonics, 2018, 12, 98.

21 H. Tanaka, K. Shizu, H. Nakanotani and C. Adachi, Chem. Mater., 2013, 25, 3766-3771.

22 Y. J. Cho, S. K. Jeon and Y. J. Lee, Adv. Opt. Mater., 2016, 4, 688-693.

23 Y. Im, M. Kim, Y. J. Cho, J. A. Seo, K. S. Yook and J. Y. Lee, Chem. Mater., 2017, 29, 1946-1963.

24 L. S. Cui, H. Nomura, Y. Geng, J. U. Kim, H. Nakanotani and C. Adachi, Angew. Chem., 2017, 129, 1593-1597.

25 C. S. Oh, D. de Sa Pereira, S. H. Han, H. J. Park, H. F. Higginbotham, A. P. Monkman and J. Y. Lee, ACS Appl. Mater. Interfaces, 2018, 10, 35420-35429.

26 K. Wang, Y. Z. Shi, C. J. Zheng, W. Liu, K. Liang, X. li, M. Zhang, H. Lin, S. L. Tao, C. S. Lee, X. M. Ou and X. H. Zhang, ACS Appl. Mater. Interfaces, 2018, 19, 3151531525.

27 Y. J. Cho, S. K. Jeon, B. D. Chin, E. Yu and J. Y. Lee, Angew. Chem., 2015, 127, 5290-5293.

28 H. Tanaka, K. Shizu, H. Nakanotani and C. Adachi, J. Phys. Chem. C, 2014, 118, 15985-15994.

29 I. Marghad, D. H. Kim, X. Tian, F. Mathevet, C. Gosmini, J. C. Ribierre and C. Adachi, ACS Omega, 2018, 3, 2254-2260.

30 D. G. Chen, T. C. Lin, Y. A. Chen, T. C. Lin, Y. T. Chen and P. T. Chou, J. Phys. Chem. C, 2018, 122, 12215-12221.

31 Y. J. Lien, T. C. Lin, C. C. Yang, Y. C. Chiang, C. H. Chang, S. H. Liu, Y. T. Chen, G. H. Lee, P. T. Chou, C. W. Lu and Y. Chi, ACS Appl. Mater. Interfaces, 2017, 9, 27090-27101.

32 D. G. Chen, T. C. Lin, C. L. Chen, Y. T. Chen, Y. A. Chen, G. H. Lee, P. T. Chou, C. W. Liao, P. C. Chiu, C. H. Chang, Y. J. Lien and Y. Chi, ACS Appl. Mater. Interfaces, 2018, 10, 12886-12896.

33 J. S. Ward, R. S. Nobuyasu, M. A. Fox, A. S. Batsanov, J. Santos, F. B. Dias and M. R. Bryce, J. Org. Chem., 2018, 83, 14431-14442.

34 K. Wang, C. J. Zheng, W. Liu, K. Liang, Y. Z. Shi, S. L. Tao, C. S. Lee, X. M. Ou and X. H. Zhang, Adv. Mater., 2017, 29, 1701476.

35 M. Okazaki, Y. Takeda, P. Data, P. Pander, H. Higginbotham, A. P. Monkman and S. Minakata, Chem. Sci., 2017, 8, 26772686.

36 P. Pander, R. Motyka, P. Zassowski, M. K. Etherington, D. Versano, T. J. da Silva, M. J. Caldas, P. Data and A. P. Monkman, J. Phys. Chem. C, 2018, 122, 23934-23942.

37 M. J. Frisch, G. W. Trucks, H. B. Schlegel, G. E. Scuseria, M. A. Robb, J. R. Cheeseman, G. Scalmani, V. Barone, B. Mennucci, G. A. Petersson, H. Nakatsuji, M. Caricato, X. Li, H. P. Hratchian, A. F. Izmaylov, J. Bloino, G. Zheng, J. L. Sonnenberg, M. Hada, M. Ehara, K. Toyota,
R. Fukuda, J. Hasegawa, M. Ishida, T. Nakajima, Y. Honda, O. Kitao, H. Nakai, T. Vreven, J. A. Montgomery Jr, J. E. Peralta, F. Ogliaro, M. Bearpark, J. J. Heyd, E. Brothers, K. N. Kudin, V. N. Staroverov, R. Kobayashi, J. Normand, K. Raghavachari, A. Rendell, J. C. Burant, S. S. Iyengar, J. Tomasi, M. Cossi, N. Rega, J. M. Millam, M. Klene, J. E. Knox, J. B. Cross, V. Bakken, C. Adamo, J. Jaramillo, R. Gomperts, R. E. Stratmann, O. Yazyev, A. J. Austin, R. Cammi, C. Pomelli, J. W. Ochterski, R. L. Martin, K. Morokuma, V. G. Zakrzewski, G. A. Voth, P. Salvador, J. J. Dannenberg, S. Dapprich, A. D. Daniels, Ö. Farkas, J. B. Foresman, J. V. Ortiz, J. Cioslowski and D. J. Fox, Gaussian 09, D.01, Gaussian, Inc., Wallingford CT, 2009.

38 S. Hirata and M. Head-Gordon, Chem. Phys. Lett., 1999, 314, 291-299.

39 M. J. Peach, M. J. Williamson and D. J. Tozer, J. Chem. Theory Comput., 2011, 7, 3578-3585.

40 T. Lu and F. Chen, J. Comput. Chem., 2012, 33, 580-592.

41 C. Wang, C. Deng, D. Wang and Q. Zhang, J. Phys. Chem. C, 2018, 122, 7816-7823.

42 J. C. Ribierre, T. Aoyama, T. Muto, Y. Imase and T. Wada, Org. Electron., 2008, 9, 396.

43 T. Förster, Ann. Phys., 1948, 437, 55.

44 A. Ruseckas, J. C. Ribierre, P. E. Shaw, I. D. W. Samuel, S. V. Staton and P. L. Burn, Appl. Phys. Lett., 2009, 95, 183305.

45 K. J. Lee, J. H. Woo, E. S. Kim, Y. Xiao, X. Su, L. M. Mazur, A. J. Attias, F. Fages, O. Cregut, A. Barsella, F. Mathevet, L. Mager, J. W. Wu, A. D'Aleo and J. C. Ribierre, Phys. Chem. Chem. Phys., 2016, 18, 7875.

46 A. D'Aleo, M. H. Sazzad, D. H. Kim, E. Y. Choi, J. W. Wu, G. Canard, F. Fages, J. C. Ribierre and C. Adachi, Chem. Commun., 2017, 53, 7003-7006.

47 K. J. Lee, Y. U. Lee, F. Fages, J. C. Ribierre, J. W. Wu and A. D'Aleo, Nano Lett., 2018, 18, 1476.

48 V. Bulovic, V. Khalfin, G. Gu, P. Burrows, D. Garbuzov and S. R. Forrest, Phys. Rev. B, 1998, 58, 3730-3740.

49 J. Huang, J. Blochwitz-Nimoth, M. Pfeiffer and K. Leo, J. Appl. Phys., 2003, 93, 838.

50 S. K. So, W. K. Choi, L. M. Leung and K. Neyts, Appl. Phys. Lett., 1999, 74, 1939.

51 W. M. V. Wan, N. C. Greenham and R. H. Friend, J. Appl. Phys., 2000, 87, 2542.

52 S. Hofle, T. Lutz, A. Egel, F. Nickel, S. W. Kettlitz, G. Gomard, U. Lemmer and A. Colsmann, ACS Photonics, 2014, 1, 968973.

53 C. Schmitz, M. Thelakkat and H. W. Schmidt, Adv. Mater., 1999, 11, 821-826.

54 W. L. Barnes, J. Mod. Opt., 1998, 45, 661-699.

55 Z. B. Wang, M. G. Helander, X. F. Xu, D. P. Puzzo, J. Qiu, M. T. Greiner and Z. H. Lu, J. Appl. Phys., 2011, 109, 053107. 56 L. Zhao, T. Komino, M. Inoue, J. H. Kim, J. C. Ribierre and C. Adachi, Appl. Phys. Lett., 2015, 106, 063301. 